\title{
Caudate Shape Discrimination in Schizophrenia Using Template-Free Non-parametric Tests
}

\author{
Y. Sampath K. Vetsa ${ }^{1}$, Martin Styner ${ }^{1}$, Stephen M. Pizer ${ }^{1}$, \\ Jeffrey A. Lieberman ${ }^{2}$, and Guido Gerig ${ }^{1,2}$ \\ 1 Department of Computer Science, \\ 2 Department of Psychiatry, \\ University of North Carolina, Chapel Hill, NC 27599, USA \\ gerig@cs.unc.edu ${ }^{\star}$
}

\begin{abstract}
This paper describes shape analysis of the caudate nucleus structure in a large schizophrenia study (30 controls, 60 schizophrenics). Although analysis of the caudate has not drawn the same attention as the hippocampus, it is a key basal ganglia structure shown to present differences in early development (e.g. autism) and also to present changes due to drug treatment. Left and right caudate were segmented from high resolution MRI using a reliable, semi-automated technique. Shapes were parametrized by a surface description, aligned, and finally represented as medial mesh structures (m-reps). Since schizophrenia patients were categorized based on treatment, we could test size and shape differences between normals, atypically and typically treated subjects. Statistical shape analysis used permutation tests on objects represented by medial representations. This allowed us to bypass the common problems of feature reduction inherent to low sample size and high dimensional feature vectors. Moreover, this test is non-parametric and does not require the choice of a shape template. The choice of medial shape representations led to a separate testing of global and local growth versus deformation. Results show significant caudate size and shape differences, not only between treatment groups and controls, but also among the treatment groups. Shape differences were not found when both treatment groups were grouped into one patient group and compared to controls. There was a clear localization of width and deformation change in the caudate head. As with other clinical studies utilizing shape analysis, results need to be confirmed in new, independent studies to get full confidence in the interpretation of these findings.
\end{abstract}

\section{Introduction}

Morphologic analysis of brain structures based on high-resolution MRI has become a common technique to assess changes and progression of changes due to

\footnotetext{
* This work was supported by the NCI Grant P01 CA47982. The Stanley Medical Research Institute is acknowledged for funding the MRI schizophrenia study providing the caudate structures. Miranda Chakos and Scott Schobel are acknowledged for providing the caudate segmentation results. Color paper version: www.cs.unc. edu/ ${ }^{\text {gerig. }}$
} 
illness, and effects of drug treatment. Studies have documented the capacity of conventional antipsychotic or neuroleptic drugs to produce volume increases in the caudate nucleus and putamen, for example 12 . These findings of caudate volume changes have been recently confirmed by Levitt et al. 3 .

Morphologic assessment of anatomical shapes increasingly embraces new analysis options provided by shape analysis methods, since they provide a rich set of features not accessible by conventional volumetry. In their seminal clinical hippocampal shape study, Csernansky et al. 4] advocate a full characterization of neuroanatomical abnormalities. Recent research in high-dimensional statistical description [5] has shown that detectable shape differences exist although the quantitative correlation of these shape differences to intuitive neuroanatomical measurements is not feasible. As an alternative to high-dimensional warping or surface-based analysis, we apply a medially-based shape representation technique introduced in our earlier work 677. Approaches for shape representation can be categorized into methods representing shapes as sets of landmarks [8], as highdimensional deformation fields 9,1011, and as single object characterizations of surfaces 121314] or skeletons [15/716]. Most shape representations characterized complex shape by high-dimensional feature vectors. Statistical shape analysis thus has to reduce this high dimensional feature space to a small set of uncorrelated features, most often using principal component analysis [12 14!9].

Localization of significant shape effects often requires a large number of statistical tests on surfaces. Correction for multiple tests, especially in the presence of local correlations across complex surfaces, has been approached by permutation tests 11] but still presents a challenging issue. Golland et al. applied permutation tests for group classification in structural and functional neuroimaging studies to assess cortical thickness and fMRI activation [17. This paper illustrates that significant feature reduction is achieved by using a sampled medial representation (m-rep). Shapes represented by coarse meshes of $3 \times 8$ medial nodes, for example, are characterized by 24 nodes with position and radius, but can be reconstructed through implied surfaces to closely approximate the original objects [7]. We also present a technique for shape discrimination that uses permutation tests on medial mesh representations. Pairwise group differences are calculated by an L1 distance between average shapes, either on the radius feature or the mesh deformation feature of the meshes. The method proposed here does not require to select a template shape against which all the shapes would have to be compared.

\section{Methods}

Clinical Study. The patient study is cross-sectional in design and assesses potential differences in brain structures between patients in the first few years of illness versus patients who have been chronically ill. Early illness (age 16-30, $\mathrm{N}=34$ ) and chronic (age 31-60, $\mathrm{N}=22$ ) patients have been matched to a young and an older control group $(\mathrm{N}=26)$. Patients were characterized with regards to duration of illness and illness severity utilizing PANSS assessments. All patients and comparison subjects were right-handed males. At the time of scan, 17 early 
illness patients were on typical antipsychotic medication (haloperidol), and 17 were on atypical antipsychotic medications (13 olanzapine, 4 on risperidone). For chronic patients, only 5 were on typical medications ( 3 haloperidol, 1 trifluperazine and 1 thiothixene), and 18 were on atypical medications (6 olanzapine, 8 clozapine and 4 risperidone). Three of the 82 total subjects had to be excluded from the analysis due to poor quality MRI data.

Shape Modeling. Caudate structures were segmented from MRI data by a semiautomated technique. Following automatic tissue classification, a trained user masks the gray matter structure of the caudate by overpainting. The technique is very efficient and reliable (intra- and inter-class reliability of 0.95 and 0.92). After segmentation, shapes were processed by an analysis pipeline that includes surface extraction and parametrization using spherical harmonics [14, object alignment using first degree spherical harmonics, creating a medial model with optimal minimal mesh sampling given the whole population [7, and m-rep model deformation into each object [16]. As a result, each object is represented by a medial mesh with fixed topology and mesh sampling. We just used the node position and the radius of the m-rep representation and not the full set of local figural frames.

Statistical Analysis by Permutation Tests. The analysis follows the well-known concept of non-parametric permutation tests. We use the method and software provided by Efron [18. In permutation tests, the difference between groups is tested against distances provided by all possible permutations of samples. This enormous task is reduced by providing a large number of randomly selected groups, here by using a Monte Carlo approach. The distance between two groups of shapes is calculated as the Euclidean distance between group average shapes. Integrated distances across the whole mesh provide global shape difference measures. The whole scheme to find the significance of shape difference between two groups of shapes is as follows:

1. Start with two groups of shapes, $A$ and $B$, with $n_{A}$ and $n_{B}$ shapes in each group respectively.

2. Define a function to calculate a scalar shape difference value between two groups of shapes.

3. Calculate the shape difference(scalar), diff_orig between $A$ and $B$.

4. Put the shapes from the two groups, $A$ and $B$, into a common basket.

5. Select $n_{A}$ shapes randomly from this basket containing all the shapes, call this group of shapes $A^{\prime}$. Call the group $n_{B}$ shapes left in the basket $B^{\prime}$.

6. Calculate the shape difference(scalar), diff_new between $A^{\prime}$ and $B^{\prime}$.

7. Note if diff_new is more than diff_orig.

8. Repeat the steps 4, 5, 6] and 7 say numtests times.

9. Calculate the number of tests for which diff_new was greater than diff_orig, say numgreater.

10. The p-value for the significance of shape difference between $A$ and $B$ is given by the fraction numgreater/numtests. 
Permutation tests on m-rep shapes. In what follows, let $s_{A}$ and $s_{B}$ be two groups of shapes, with $n_{A}$ and $n_{B}$ shapes in each group, respectively. Let a simplified m-rep model be given by $\left[\left\{\mathbf{x}_{k}, r_{k}\right\} \mid k \in\{r \times c\}\right]$, where $k$ is the $\mathrm{k}$-th node of a mesh of size $(r \times c), \mathbf{x}_{k}$ is the vector of the $3 \mathrm{D}$ coordinates of the $\mathrm{k}$-th node, and $r_{k}$ is the radius of the $\mathrm{k}$-th node. Let $\mathbf{x}_{k i}$ and $r_{k i}$ represent the node locations and radii for the $\mathrm{i}$-th shape.

Given a group of m-reps, the location of an atom in the average m-rep is defined as the average of locations of that atom in all the m-reps in the group: $\mathbf{x}_{k-a v g}=\frac{1}{n_{A}} \sum_{i=1}^{n_{A}} \mathbf{x}_{k i}$. We assume correspondence of m-rep nodes with same mesh indices.

1. When performing the permutation test for the location of an individual atom, the shape difference between the two groups is defined as the distance between the locations of the corresponding atom in the average shapes of the two groups: $\Delta x_{k}=\left|\mathbf{x}_{k-a v g}\left(s_{A}\right)-\mathbf{x}_{k-a v g}\left(s_{B}\right)\right|_{1}$. A one-sided permutation test is used.

2. When performing the permutation test for the atom locations for the whole shape, the difference between the two groups is calculated by summing difference at each atom: $\Delta x=\sum_{k=1}^{r \times c} \Delta x_{k}$. A one-sided permutation test is used.

We use similar methods for the permutations tests for the m-rep atom radii and the volumes. For the radius test at an individual node, we take the signed difference between the average radii of the two groups at that node, and use a two-sided test. For the radius test for the whole shape, we sum the absolute differences at individual nodes and use a one-sided test. For the volume test, we take the signed difference in the average volumes of the groups and use a two-sided test.

\section{Results}

Volume analysis. Table 1 shows results of the statistical analysis for the caudate volumes, with individual normalization for the size of the intracranial volume (ICV). All tests were significant and suggest that the caudate volume indeed might reflect changes due to treatment.

Table 1. Statistics for caudate volumes, with correction for ICV (intracranial volume). We used 100,000 permutations and two-sided tests. The table lists p values.

\begin{tabular}{|c|c|c|c|c|}
\hline & SZ vs CNTL & Typ vs CNTL & Atyp vs CNTL & Typ vs Atyp \\
\hline Left & $0.00083^{* *}$ & $0.00001^{* *}$ & $0.03037^{* *}$ & $0.01493^{* *}$ \\
\hline Right & $0.00398^{* *}$ & $0.00002^{* *}$ & $0.05228^{* *}$ & $0.04111^{* *}$ \\
\hline
\end{tabular}


Global shape differences. Figure 1 shows the comparison of the mean shapes for the control, typically treated, and atypically treated groups as overlaid threedimensional figures. The figures suggest that there are differences between the groups. However, it also shows clear limitations of such type of graphical displays to get an intuitive understanding of shape changes.
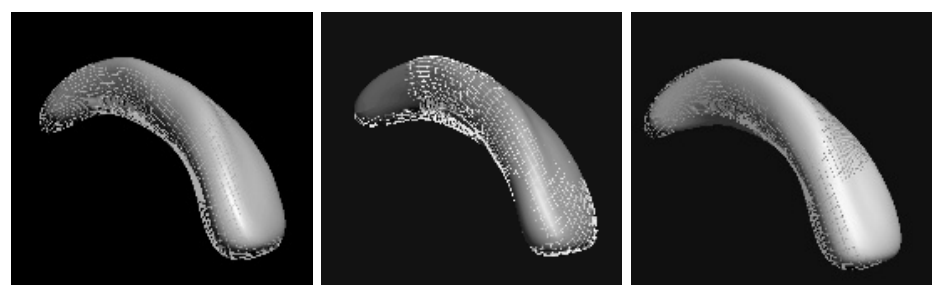

Controls vs Typicals Controls vs Atypicals Atypicals vs Typicals

Fig. 1. Comparison of mean shapes between groups. Left: Solid for controls and mesh for typicals. Middle: Solid for controls and mesh for atypicals. Right: Solid for atypicals and mesh for typicals

Table 2 lists the m-rep statistics for deformation and width, integrated over the whole shape. Results marked with ${ }^{* *}$ were found to be significant. The tables demonstrate that shape differences were found between the treatment groups, a result which is potentially very important for drug studies.

Table 2. Statistical analysis of m-rep representations (p values). Top: Mesh deformation distance (L1) calculated as the integrated deformation over the whole mesh. Bottom: Width (radius) change measure calculated as integrated absolute radius differences over the whole mesh. We used 10,000 permutations with one-sided tests.

\begin{tabular}{|c|c|c|c|c|}
\hline \multicolumn{5}{|c|}{ M-rep statistics: Deformation measure } \\
\hline & SZ vs CNTL & Typ vs CNTL & Atyp vs CNTL & Typ vs Atyp \\
\hline Left & 0.5112 & 0.0651 & 0.5781 & $0.0188^{* *}$ \\
\hline Right & 0.5742 & 0.1518 & 0.5272 & 0.07 \\
\hline
\end{tabular}

\begin{tabular}{|c|c|c|c|c|}
\hline \multicolumn{5}{|c|}{ M-rep statistics: Local Width Measure } \\
\hline & SZ vs CNTL & Typ vs CNTL & Atyp vs CNTL & Typ vs Atyp \\
\hline Left & 0.4326 & 0.0522 & 0.7581 & $0.001^{* *}$ \\
\hline Right & 0.4121 & 0.1202 & 0.4424 & $0.0022^{* *}$ \\
\hline
\end{tabular}

Localization of shape effect. Locality of shape effect is shown for the comparison between the two treatment groups, Typ versus Atyp, as this group difference was shown to be significant in the global shape test presented above.

Figure 2] shows the m-rep statistics for the local deformation, calculated using a one-sided permutation test at each node. The analysis demonstrates that 
significant deformation occurs mostly at two regions next to the middle body part and is located at the periphery of the structures.
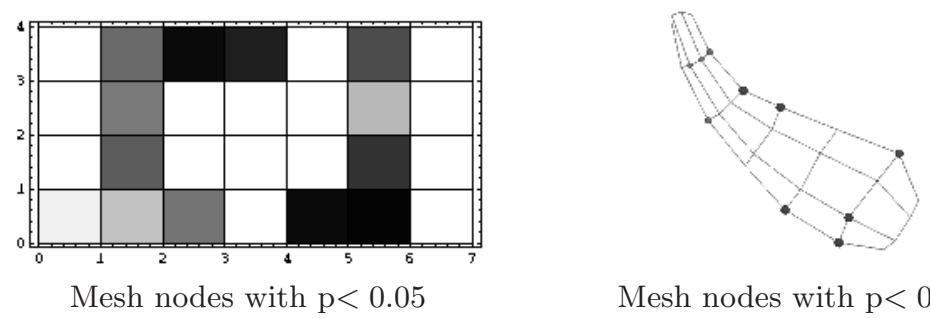

Mesh nodes with $\mathrm{p}<0.05$

Fig. 2. Local deformation statistics for Typ-Atyp group tests for the left caudate: Left: Locations on the mesh with p-values less than 0.05 are suppressed, with lower p-value shown as darker shading. Right: Locations on the m-rep mesh with p-values less than 0.05 suppressed, with the size of the ball at each node indicating the significance.

Figure 3 shows the m-rep statistics for the local width (radius), calculated using a two-sided permutation test at each node. The figure demonstrates that significant width differences are mostly found in the caudate head region.

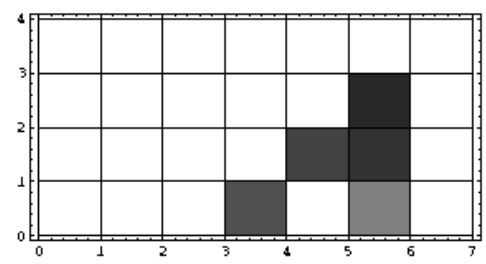

Mesh nodes with $\mathrm{p}<0.05$

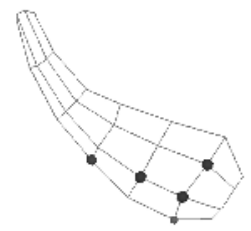

Mesh nodes with $\mathrm{p}<0.05$

Fig. 3. Local width statistics for Typ-Atyp group tests shown for the left caudate: Left: Locations on the mesh with p-values less than 0.05 are suppressed, with lower p-value shown with darker shading. Right: Locations on the m-rep mesh with p-values less than 0.05 suppressed, with the size of the ball at each node indicating the significance.

Surface display of locality of Radius difference. The significant differences of the m-rep statistical analysis can be illustrated as effects on the implied boundaries. We chose the significant Radius differences between Typical and Atypical for this comparison. Figure 4 shows the comparison between the mean shape of the atypical group and the differential added by the significant radius differences between typical and atypical as reported in Fig. 3. The figures reveals that the major change of significant radius differences is found in the caudate head. The typical group presents an enlarged head structure in comparison to the atypical group. These figures also demonstrate that our medial representation allows us 
to "stabilize" a figure (here deformation) and only ask the question for locality or radius difference at corresponding nodes, for example.

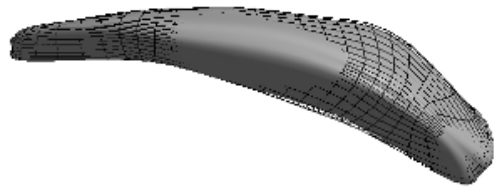

Side View

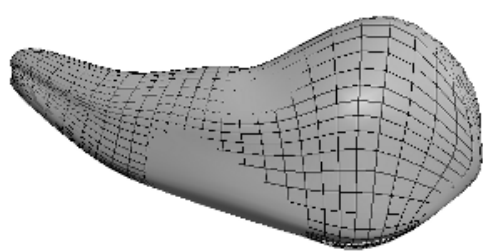

Top View

Fig. 4. Surfaces implied from m-reps. The figures show the m-rep surfaces for the average Atyp (solid) and average Atyp $+\Delta(\text { Typ }- \text { Atyp })_{\text {radius }}$ overlaid (mesh). Please note that this display differs from an overlay of average shapes and illustrates significant changes (here radius only) between groups.

\section{Discussion}

This paper discusses the application of permutation tests for shape analysis. The non-parametric test overcomes limitations of feature reduction or feature selection applied to shapes represented by a high-dimensional set of features. Instead, we calculate a scalar shape distance measure for each group difference tested in the iterative process. Shapes are represented by sampled medial meshes (m-reps). We performed separate statistical tests for mesh deformation (Euclidean distance between corresponding mesh nodes) and for local width change (Radius difference). The method proposed here also has the advantage that it does not need a template for providing shape distance measurements. Templates, especially when taken from the same population or calculated as averages of the same populations, would introduce a statistical bias. We did not yet apply correction for multiple statistical tests. Ongoing work will make use of the framework provided by the SnPM package that takes into account correlations between neighboring grid cells. As each mesh node represents a portion of the surface and of the volume, we are also developing advanced concepts for introducing appropriate weights to nodes prior to the statistical tests.

The technique is applied to a study of caudate shape in schizophrenia. Volume differences between all groups were highly significant, suggesting that the caudate volume might show changes due to treatment. Shape analysis after size normalization provides features not reflecting size changes. The findings suggest a significant shape difference between groups treated with atypical and groups treated with typical drugs, which might be either a treatment effect or a patient selection bias. The tests between controls and typical and also controls and atypical are not significant. However, they show a trend that the caudate shape of the typical group shows a larger difference than the shape of the atypical group, 
mostly on the left side. The tests not only give global shape difference results but also localization. Our finding that the major change occurs mostly in the head of the caudate might lead to a better understanding of disease progress. Significant shape differences between the treatment groups might become very interesting for pharmaceutical studies as they might lead to a better insight into effects of various drug treatments.

\section{References}

1. Lieberman, J., Jody, D., Geisler, S., Alvir, J., Loebel, A., Szymanski, S., Woerner, M., Borenstein: Time course and biological correlates of treatment response in first episode schizophrenia. Arch of Gen Psychiatry 50 (1993) 369-376

2. Chakos, M., J.A., L., Alvir, J., Bilder, R., Ashtari, M.: Caudate nuclei volumes in schizophrenic patients treated with typical antipsychotics or clozapine. Lancet (1995) 345-357 letter.

3. Levitt, J., McVarley, R., Dickey, C., Voglmaier, M., Niznikiewicz, M., Seidman, L., Hirayasu, Y., Ciszewski, A., Kikinis, R., Jolesz, F., Shenton, M.: MRI study of caudate nucleus volume and its cognitive correlates in neuroleptic-naive patients with schizotypal personality disorder. Am J Psychiatry 159 (2002) 1190-1197

4. Csernansky, J., Joshi, S., Wang, L., Haller, J., Gado, M., Miller, J., Grenander, U., Miller, M.: Hippocampal morphometry in schizophrenia via high dimensional brain mapping. Proc. Natl. Acad. Sci. USA 95 (1998) 11406-11411

5. Wang, L., Joshi, S., Miller, M., Csernansky, J.: Statistical analysis of hippocampal asymmetry in schizophrenia. NeuroImage (2000)

6. Gerig, G., Styner, M., M., S., , Lieberman, J.: Shape versus size: Improved understanding of the morphology of brain structures. In: Medical Image Computing and Computer-Assisted Intervention MICCAI 2002. Volume 2208 of LNCS., Springer Verlag (2001) 24-32

7. Styner, M., Gerig, G.: Three-dimensional medial shape representation incorporating object variability. In: Proc. of Computer Vision and Pattern Recognition CVPR 2001, IEEE Computer Society (2001) 651-656

8. Bookstein, F.: Shape and the Information in Medical Images: A Decade of the Morphometric Synthesis. CVIU 66 (1997) 97-118

9. Joshi, S., Miller, M.I.: Landmark matching via large deformation diffeomorphisms. IEEE Transactions on Image Processing 9 (2000) 1357-1370

10. Davatzikos, C.: Spatial transformation and registration of brain images using elastically deformable models. CVIU 66 (1997) 207-222

11. Thompson, P., Giedd, J., Woods, R., MacDonald, D., Evans, A., Toga, A.: Growth patterns in the developing brain detected by using continuum mechanical tensor maps. Nature 404 (2000) 190-193

12. Cootes, T., Taylor, C., Cooper, D., Graham, J.: Active shape models - their training and application. Computer Vision and Image Understanding 61 (1995) 38-59

13. Staib, L., Duncan, J.: Model-based Deformable Surface Finding for Medical Images. IEEE TMI 15 (1996) 1-12

14. Kelemen, A.and Székely, G., Gerig, G.: Three-dimensional model-based segmentation. IEEE TMI 18 (1999)

15. Golland, P., Grimson, W.: Fixed topology skeletons. In: ICCV. (1999) 
16. Pizer, S.M., Chen, J.Z., Fletcher, P.T., Fridman, Y., Fritsch, D.S., Gash, A.G., Glotzer, J.M., Jiroutek, M.R., Joshi, S., Lu, C., Muller, K.E., Thall, A., Tracton, G., Yushkevich, P., Chaney, E.L.: Deformable M-Reps for 3D Medical Image Segmentation. IJCV 55 (2003)

17. Golland, P., Fischl, B.: Permutation tests for classification towards statistical significance in image-based studies. In Taylor, C., Noble, J.A., eds.: Information Processing in Medical Imaging IPMI. LNCS 2732, Springer Verlag (2003) 330-341

18. Efron, B.: An Introduction to the Bootstrap. Chapman \& Hall (1998) Download Mathematica Software Permutation Tests:

http://library.wolfram.com/infocenter/MathSource/. 